\title{
Multifaced pknE: Apoptosis Inhibition, HIV Co-Infection, Host Signaling Cross-Talk and in Orchestrating the Physiology of Mycobacterium tuberculosis
}

\author{
Dinesh Kumar Parandhaman ${ }^{1,2}$, Sameer Hassan ${ }^{3}$ and Sujatha Narayanan ${ }^{1^{*}}$ \\ ${ }^{1}$ Department of Immunology, National Institute for Research in Tuberculosis Research, Chennai, India \\ ${ }^{2}$ Department of Immunology, International Centre for Genetic Engineering and Biotechnology, Aruna Asif Ali Marg, New Delhi, India
}

${ }^{3}$ Department of Biomedical Informatics, National Institute for Research in Tuberculosis Research, Chennai, India

\begin{abstract}
Serine/threonine protein kinases (STPK) regulate various functions in the pathogenesis of Mycobacterium tuberculosis and are listed as prime targets for the cure of tuberculosis (TB) disease. Genetic deletion of $p k n E$ helped to unravel its role in nitric oxide stress, an important antimicrobial agent produced by host cells. pknE is well characterized for its functions in host as well as in M. tuberculosis physiology. The current review summarizes the multiple roles of $p k n E$ in human pathogenesis. pknE remains the only STPK that has the standalone function of apoptosis suppression and probable role in HIV co-infection.
\end{abstract}

Keywords: Mycobacterium tuberculosis; Pathogenesis; Mycobacterial physiology

\section{Introduction}

Genomic studies have identified numerous signaling networks within Mycobacterium tuberculosis (M. tuberculosis), the causative organism of tuberculosis (TB) [1]. The unusual presence of eukaryotic like serine/threonine/tyrosine protein kinases and their phosphatases in $M$. tuberculosis suggests the various survival mechanisms employed by pathogens to manipulate the host machinery for its survival and persistence. Emerging evidence on the characterizations of the serine/threonine protein kinases (STPK) suggests a functional superiority over two component systems in M. tuberculosis pathogenesis. The 11 STPKs of M. tuberculosis have four predominant functions cell division ( $p k n A, p k n B, p k n F, p k n L)$ [2-4], intracellular survival ( $p k n E, p k n G, p k n H, p k n I$ and $p k n K)$ [59], apoptosis suppression ( $p k n E)$ [6] and host adaptations $(p k n H$, $p k n E, p k n F, p k n G)[8,10-12]$. In addition, the 11 STPKs were found to have shared substrates as observed in eukaryotic systems [1]. In this review we share our experience in analyzing $p k n E$, the only gene from STPK family that has been functionally characterized both in host and mycobacterial physiology.

\section{Molecular Signature of $p k n E$}

pknE was annotated to be a transporter due to the presence of neighboring genes narK2, Rv1739c and RV1747 with transporter functions [13]. The protein architecture of pknE contains intracellular $\mathrm{N}$ - terminal kinase domain, transmembrane domain followed by extracellular C-terminal domain [14]. Crystallographic studies [15] , and our in silico analysis (un published data) revealed the presence of CXXC motif in the periplasmic C-terminal region. CXXC motif occupies the active sites of thioredoxin superfamily members suggesting a redox function for pknE [15]. However, the importance of the CXXC motif in the pknE mediated functions remains to be studied. Phylogenetic classification placed $p k n E$ under the family of integral membrane receptor and cytoplasmic kinases [16].

\section{Paralogs of pknE and the Clue to Pathogenesis}

pknE was cloned and over expressed for biochemical studies. Purified protein had kinase activity that was dependent on the metal ions $\mathrm{Mn}^{2+} / \mathrm{Mg}^{2+}$ [6]. Sprotein purification studies by [14] observed the truncation of $\mathrm{C}$ - terminal region, suggesting secretion of pknE. Though we observed a similar proteolytic product, this was not confirmed by mass spectrometry.

Promoter identification studies using gene trap vector system suggested a putative promoter to lie within 545 bp upstream to the $p k n E$ gene. Gene regulation analysis under varying stress conditions were carried out using $M$. smegmatis as the surrogate host. These studies highlighted the putative promoter to respond heat, nutrient deprivation and nitrate stress [6]. The finding from our promoter studies that $p k n E$ responds NO stress well synchronized with the functional data of its paralogs from cyanobacterium Synechocystis and Anabaena $[17,18]$ where it regulates nitrogen fixation. Molecular pathogenesis studies were carried out by generating a deletion mutant of $p k n E(\Delta p k n E)$ as reported earlier [19].

\section{Role in Apoptosis}

In a macrophage model of infection, deletion of $p k n E$ resulted in reduced intracellular survival with parallel increase in macrophage cell death [6]. Analyses of cell death phenotypes showed that $\Delta p k n E$ infected macrophages undergo apoptotic cell death as confirmed by TUNEL assay. The role for necrosis mediated cell death was ruled out using LDH assay measurements. Interestingly, the increased apoptosis observed in $\Delta p k n E$ infected macrophages did not increase the pro-inflammatory cytokines. To the best of our knowledge, $p k n E$ still retains the only STPK that suppresses apoptosis.

*Corresponding author: Sujatha Narayanan, Department of Immunology, National Institute for Research in Tuberculosis Research, Chennai, India, Tel: +9144-28369627; E-mail: sujatha.sujatha36@gmail.com

Received May 25, 2016; Accepted June 07, 2016; Published June 14, 2016

Citation: Parandhaman DK, Hassan S, Narayanan S (2016) Multifaced pknE: Apoptosis Inhibition, HIV Co-Infection, Host Signaling Cross-Talk and in Orchestrating the Physiology of Mycobacterium tuberculosis. J Microb Biochem Technol 8: 231-235. doi: 10.4172/1948-5948.1000291

Copyright: ( 2016 Parandhaman DK, et al. This is an open-access article distributed under the terms of the Creative Commons Attribution License, which permits unrestricted use, distribution, and reproduction in any medium, provided the original author and source are credited. 
Citation: Parandhaman DK, Hassan S, Narayanan S (2016) Multifaced pknE: Apoptosis Inhibition, HIV Co-Infection, Host Signaling Cross-Talk and in Orchestrating the Physiology of Mycobacterium tuberculosis. J Microb Biochem Technol 8: 231-235. doi: 10.4172/1948-5948.1000291

\section{pknE Suppresses Intrinsic Apoptosis}

Apoptosis or cell death can be executed by various paradigms and M. tuberculosis was shown to suppress wide array of apoptotic mechanisms and only few genes were identified in this responses [20]. Our microarray based approach to study the function of $p k n E$ in modulating the immune responses of the host macrophages revealed its role in suppression of mitochondrial apoptosis besides TP53 mediated cell death. Microarray data showed Bax, Bid (mitochondrial proteins), arginase2, caspase-9, TP53 to be increased in the $\Delta p k n E$ infected macrophages compared to its wild type H37Rv [21].

Infection of $\Delta p k n E$ with THP-1 macrophages activated wide array of Toll like receptors 2, 4, 6, 8 and 9 suggesting stronger host cell activation [21]. In addition, $\Delta p k n E$ infected macrophages had increased $\beta$-chemokine secretion and reduced expression of the co stimulatory molecules CD80/CD86 [21]. Microarray based studies also validated that $\triangle p k n E$ infected macrophages have reduced proinflammatory cytokines and iNOS expression. RNA based studies validated that $\triangle p k n E$ infected macrophages undergo TNF, iNOS and caspase-8 independent apoptosis.

\section{pknE Modulates the MAPK Signaling for M. tuberculosis Survival}

Mitogen-activated protein kinases (MAPKs) regulate multiple physiological responses in eukaryotes including apoptosis and cytokine production [22]. MAPKs comprises the conventional MAPKs extracellular signal-regulated kinases $1 / 2(\mathrm{ERK} 1 / 2)$, c-Jun amino $(\mathrm{N})$ terminal kinases $1 / 2 / 3(\mathrm{JNK} 1 / 2 / 3)$, p38 isoforms $(\alpha, \beta, \gamma$ and $\delta)$, ERK5 and the atypical kinases [22]. Modulation of MAPK signaling was suggested a survival strategy by the virulent strains of $M$. tuberculosis [23]. Analysis of the MAPK signaling was of prime importance since $\Delta p k n E$ infected macrophages increased apoptosis with decrease in pro inflammatory cytokines. Furthermore, purified pknE cross reacted with SAPK/JNK antibody among the MAPKs tested $[6,21,24]$. Analysis of the MAPK phosphorylation kinetics showed $\triangle p k n E$ to reduce the phosphorylation of Erk1/2, p38MAPK and selectively inhibiting the phosphorylation of $\mathrm{p} 46$ subunit of SAPK/JNK post infection compared to Rv infected macrophages [24]. Subsequent phospho kinetic analysis of the transcription factors ATF-2 and c-JUN the downstream targets for the MAPK signaling revealed a similar decrease in their activation. This emphasized that deletion of $p k n E$ reduces the phosphorylation kinetics of MAPK signaling that is well supported with decreased pro-inflammatory cytokine secretion. In addition, similar reduction in the pro-survival Akt signaling was exhibited by $\Delta p k n E$ infected macrophages [21]. These findings were analyzed using pathway specific inhibitors. Paradoxically, the pathway specific inhibitors p38MAPK, Erk1/2, SAPK/JNK or Akt were unable to suppress the MAPK activation in the $\triangle p k n E$ infected macrophages which was observed in its wild-type strain. This prompted the occurrence of crosstalk signaling in macrophages infected with $\Delta p k n E$. Our crosstalk studies showed that, inhibition of Erk1/2 pathway did not affect the phosphorylation of SAPK/JNK while inhibition of SAPK/JNK pathway by its specific inhibitor reduced the phosphorylation of Erk1/2 in $\Delta p k n E$ infected macrophages as compared to its wild type strain [24]. Further, we wanted to dissect the MAPK that could be involved in this crosstalk. We used siRNA approach to knockdown the SAPK/JNK pathway in THP-1 macrophages. Knock down of JNK1 (JNK46) did not affect the phosphorylation of SAPK/JNK or Erk1/2 neither in $\Delta p k n E$ infected macrophages or its wild type infection. Interestingly, knock down of JNK2 (p54SAPK) reduced the phosphorylations of SAPK/JNK and
Erk1/2 in macrophages infected with $\Delta p k n E$ when compared to Rv infected macrophages (un published data). This shows that $\Delta p k n E$ uses JNK2 to reduce the Erk1/2 signaling.

Collectively the signaling studies highlight that deletion of $p k n E$ renders the mutant bacilli to decrease the activation of pro-survival kinases Erk1/2 and Akt by crosstalk that costs the decreased survival of both $M$. tuberculosis and infected macrophages. Further animal studies using MAPK knock outs would decipher the significance of these in vitro observations.

\section{pknE in Tolerance to Nitric Oxide (NO) Stress}

Production of reactive nitrogen species (RNS), reactive oxygen species (ROS) is an important host defense mechanism to protect against the invading pathogen. iNOS is an important enzyme involved in nitric oxide (NO) production. M. tuberculosis was shown to survive NO stress response [25]. To better understand the functions of $p k n E$ in host NO stress, we used NO donor sodium nitro prusside that mimics in vivo situations of NO stress in a macrophage model [26]. Induction of NO stress revealed $\Delta p k n E$ infected macrophages to have similar host phenotypes that was observed in the absence of the NO donor [21]. $\Delta p k n E$ infected macrophages underwent increased apoptosis that was dependent on Bax, arginase 2, caspase- 9 and TP53. This data highlighted that the mutant is unable to survive NO stress and succumbs to the host apoptosis implicating a role for $p k n E$ in NO stress. Estimation of the levels of NO using griess assay showed modest difference between $\Delta p k n E$ versus its wild type $\mathrm{H} 37 \mathrm{Rv}$. Furthermore, the expression analysis of iNOS by qRT-PCR failed to show any transcripts levels post infection in the presence of NO stress, while a decrease was observed in the absence of NO stress [6,21]. These differences compelled us to examine the arginase metabolism, a component of urea cycle that is involved in NO production. During urea cycle iNOS is involved in the conversion of L-arginine to NO, and L-citrulline [27]. Within the urea cycle, arginase also shares the substrate $\mathrm{L}$-arginine for the production of ornithine and urea. We analyzed the genes arginase1, arginase2, arginosuccinate synthase and arginosuccinate lyase.

Both in the presence and absence of $\mathrm{NO}$ donor $\Delta p k n E$ infected macrophages had reduced expression of arginase1 with increase in arginase2 [21]. Expression of arginase 1 was reported to be involved in virulence strategies of $M$. tuberculosis [28]. Our arginase assay during NO stress also proved the reduction in arginase 1 and increase in arginase 2. The results from arginase metabolism favor the expression of arginase2 over iNOS and this in turn supports apoptosis of the $\Delta p k n E$ infected macrophages. This feature is similar to that observed in Helicobacter pylori infection [29].

We also analyzed the MAPK and Akt signaling during NO stress. $\triangle p k n E$ infected macrophages reduced the phosphorylation of Akt, Erk1/2 and SAPK/JNK while an increase in p38 was found $[21,24]$. These findings were similar to that observed in the absence of NO donor. Using exogenous NO donor, we conclude that deletion of $p k n E$ renders the mutant bacilli susceptible to host immunity. $p k n E$ mediated modulations increase the host cell survival pathways that mutually benefits the host and $M$.tuberculosis albeit compromising the immunity of the host.

\section{pknE Protects the Cellular Integrity of M. tuberculosis During Host Stress}

Macrophage studies using $\Delta p k n E$ highlighted that $p k n E$ is involved in the suppression of apoptosis. $\Delta p k n E$ was examined for 
Citation: Parandhaman DK, Hassan S, Narayanan S (2016) Multifaced pknE: Apoptosis Inhibition, HIV Co-Infection, Host Signaling Cross-Talk and in Orchestrating the Physiology of Mycobacterium tuberculosis. J Microb Biochem Technol 8: 231-235. doi: 10.4172/1948-5948.1000291

phenotypic variations and its survival to various stress conditions that are encountered inside the host. Independent survival experiments performed using $\mathrm{pH}$ and surfactant stress showed $\Delta p k n E$ to have better survival than its wild-type strain. However, the growth of $\Delta p k n E$ was markedly reduced when exposed to both the stress conditions simultaneously [12]. This validates the inability of $\Delta p k n E$ to survive multiple stresses encountered within the phagosome and compliments our data of reduced intracellular survival that we found in macrophages [6]. In addition, we found that absence of Tween 80 in cultures induces increased cell aggregation in $\Delta p k n E$. However, this increased cell aggregation did not show major defects in the bio-film formation experiments when $\Delta p k n E$ was compared to its wild type strain [12]. Morphological analysis of the $\Delta p k n E$ displayed altered cell size during growth in Middlebrook $7 \mathrm{H} 9$ while morphological abnormalities were observed only during biofilm formation as compared to its wild type. Other phenotypic factors like IS6110 finger print, Ziehl Neelson staining, and mycolic acid analysis did not show any differences between $\Delta p k n E$ and its wild type. Interestingly, $\Delta p k n E$ exhibited kanamycin resistance, a second line TB drug. Sequencing analysis of the rrs gene of either wildtype or $\Delta p k n E$ did not show any differences suggesting a difference in the expression of efflux pumps. The survival of $\Delta p k n E$ was assessed in a guinea pig model, where it exhibited hyper virulence [12] similar to that of $\Delta p k n H$ [8]. It is well documented that strains that induce increased apoptosis produce hyper virulence in animal models. Though $p k n H$ and $p k n E$ share the phenomenon of hyper virulence in animal models, the other characteristics for $p k n H$ is currently unknown. Collectively these results suggest that $p k n E$ plays a role in adaptive response of $M$. tuberculosis in regulating cell integrity and survival during host stress.

\section{Survival Strategies of $p k n E$ can Impact HIV Infection}

Tuberculosis was shown to increase HIV replication that accelerates the progression of HIV infection [30]. Similarly, HIV co-infection activates latent $\mathrm{TB}$ infection to the progression of the active TB disease [31]. Though wide reports both in the pathogenesis and epidemiological contexts exist, the genetic determinants from $M$. tuberculosis that could be involved in this co-infection are unknown. $\triangle p k n E$ was an attractive model to study the consequence of TB on promoting HIV replication due to its role in innate immunity as described in earlier sections. CCR5 and CXCR4, the co-receptors involved in HIV entry was investigated upon infection with $\triangle p k n E$ and its wild-type strain. Decreased CCR5 and increased CXCR4 levels were observed upon $\triangle p k n E$ infection, which was further validated in an in vitro model of co-infection [24]. We extended our findings by analyzing the impacts of MAPK, Akt and arginase signaling in the co-infection. SAPK/JNK and arginase signaling had a prominent role in the modulation of these co-receptors and our in vitro co-infection model authenticated the role of SAPK/ JNK signaling during HIV-TB co-infection. It is evident from the above findings that $M$. tuberculosis survival strategies can provide niche for the progression of HIV co infections and this can impact the survival of the host.

\section{Proteomics Studies Suggest pknE Could Regulate sigB Responsive Genes}

We endeavored to analyze the substrates for PknE due to its role in innate immunity using both in silico and in vivo proteomic studies. Numerous substrates were identified for $M$. tuberculosis STPKs including $p k n E$ [1]. However, the substrates were found to be commonly shared between the STPKs, though functional differences were observed. Our initial in silico analysis was based on the sequences from the activation loop that were identified in an earlier study [32].
This method was also used to identify substrates for $\mathrm{PknH}$ [33]. Since the substrate binding clefts for $\mathrm{pknE}, \mathrm{pknD}$ and $\mathrm{pknH}$ have structural similarities, this analysis did not identify any novel substrates for pknE (our unpublished data). When we included CXXC motif present in the $\mathrm{C}$ - terminal of the protein, novel substrates were identified and was validated using predikin database. Substrates with higher scores are depicted in Figure 1. Besides, the approaches used by us, homologous protein mapping model that encompasses data from experimentally validated substrates and STRING database suggested substrates for pknE $[34,35]$. However, these in silico predictions await experimental validation.

Our in vivo proteomic studies using $2 \mathrm{D}$ - gel electrophoresis and mass spectrometry highlighted various roles for $p k n E$. Substrates were identified by comparing the proteomes derived from $\Delta p k n E$ and its wild type grown in Middlebrook 7H9 and in the presence of NO stress using sodium nitro prusside. PknE was found to play a role in cell division, virulence, dormancy, suppression of sigma factor B and its regulated genes, suppression of two-component systems and in the metabolic activity of $M$. tuberculosis [36]. Functional attributes of PknE substrates were already depicted with a working model in earlier report [36]. It is noteworthy that the substrates PstP and Wag31 identified as substrates for PknE by us using in vivo proteomic approach was predicted as probable substrate in a in silico based study [35].

\section{Conclusion}

Our findings from the host responses by which $p k n E$ could modulate the host immunity are depicted as a model in Figure 2. Though findings

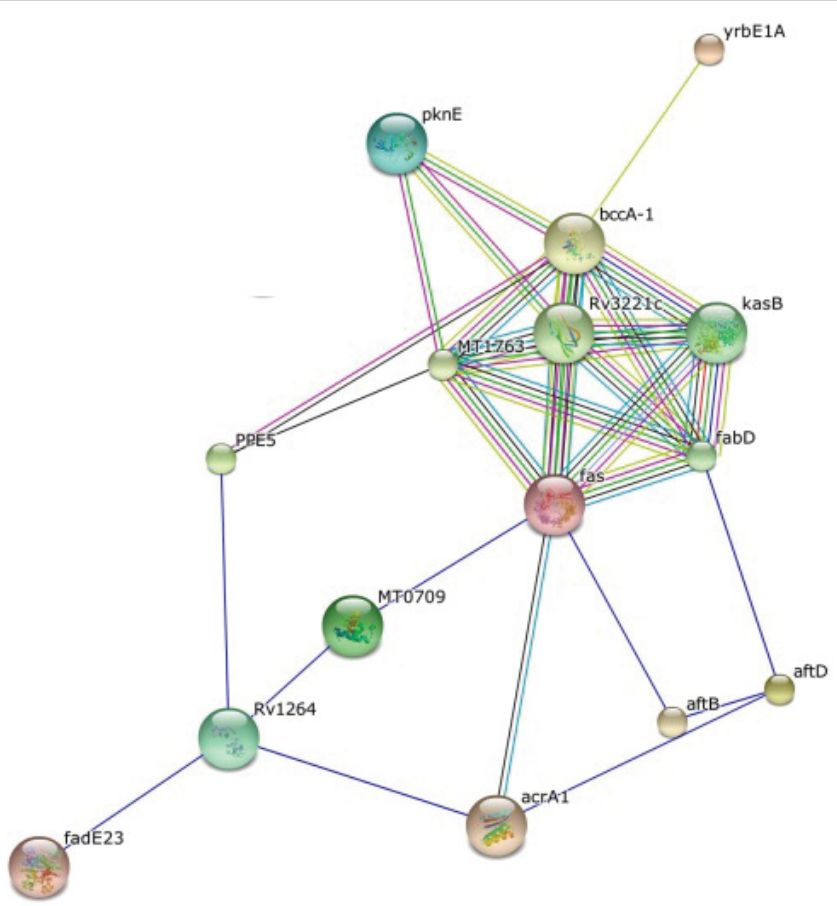

Figure 1: Cytoscape image depicting the putative substrates for PknE. This figure summarizes the predicted associations for PknE with the group of proteins from Mycobacterium tuberculosis. Each node is a protein provided with gene name. The colored lines represent the existence of evidence used in predicting the associations. A red line indicates the presence of fusion evidence; a green line - neighbourhood evidence; a blue line - co-ocurrence evidence; a purple line - experimental evidence; a yellow line - text mining evidence; a light blue line - database evidence; a black line - co-expression evidence. 
Citation: Parandhaman DK, Hassan S, Narayanan S (2016) Multifaced pknE: Apoptosis Inhibition, HIV Co-Infection, Host Signaling Cross-Talk and in Orchestrating the Physiology of Mycobacterium tuberculosis. J Microb Biochem Technol 8: 231-235. doi: 10.4172/1948-5948.1000291

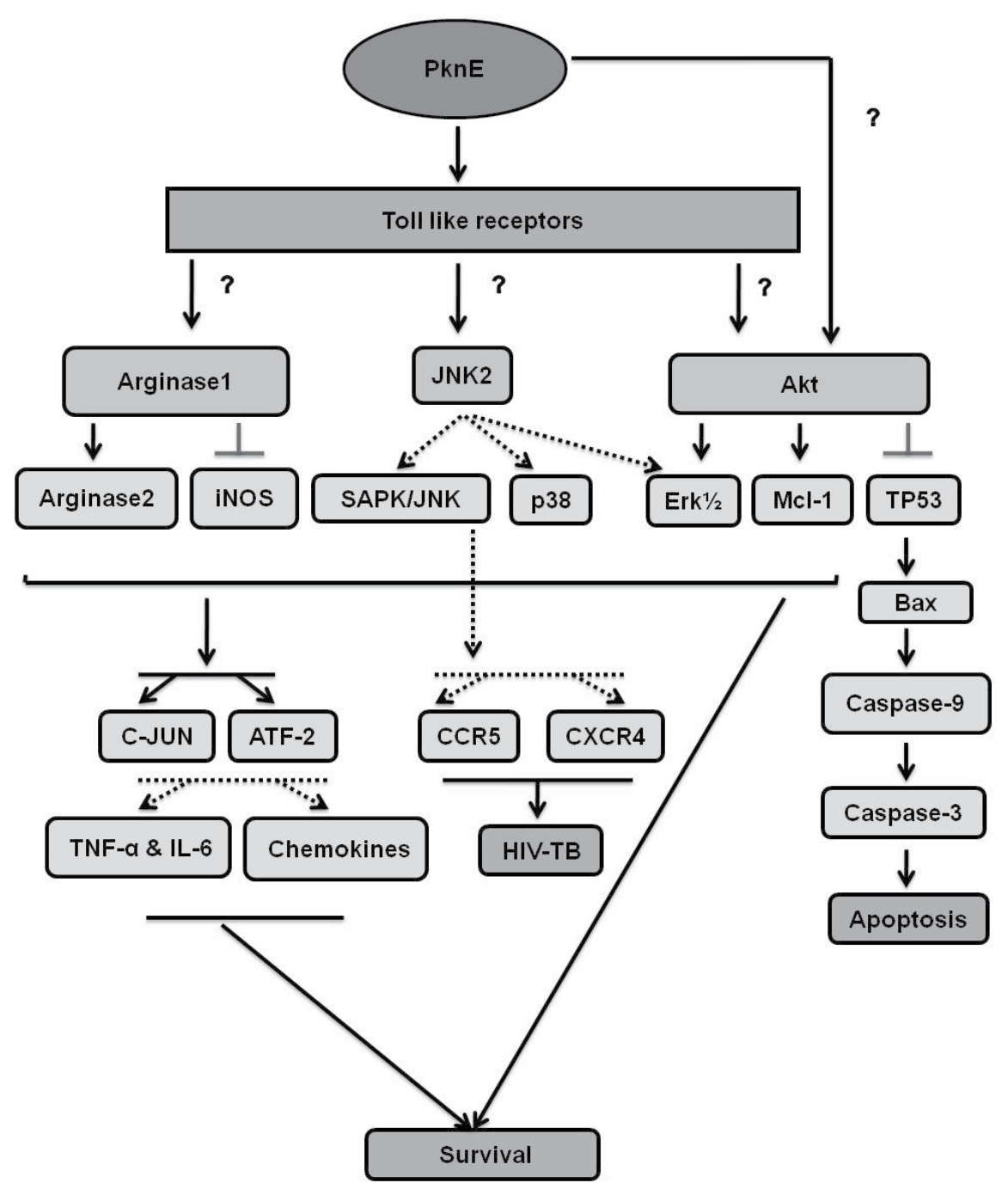

Figure 2: Illustration showing proposed pathway by which pknE could suppress innate immunity responses.

The mechanism by which PknE activates TLR, Arginase1, Akt, and JNK2 is currently unknown. JNK2 is presumably involved in MAPK cross-talks and the modulation is depicted as dotted lines. Changes in the intracellular signaling suppress apoptosis, shift the balance between cytokine vs. chemokines, and enhance HIV infection. This altered immunity helps the survival of $M$. tuberculosis. Dotted lines indicate modulation and the sign perpendicular indicates inhibition.

from our group has identified and validated multiple functions for the gene $p k n E$, few unexplored functions need experimental evidence and we hope that these would be addressed in the future. The prediction of $p k n E$ as a transporter due to the flanking of genes with transporter function warrants further studies. Though $p k n E$ could not be fully secreted due to the presence of transmembrane domain, effect of truncation of c-terminal end of PknE that contains the DsbA domain in the pathogenesis needs to be studied. The pathways by which $p k n E$ or in general the interaction of STPKs with the host pathogen detection receptors also require experimental evidence. Gaining insights in to these mechanisms would enable us to know how pathogens knock down immunity and this can have impacts on new therapeutics in the forthcoming years.

\section{References}

1. Chao J, Wong D, Zheng X, Poirier V, Bach $\mathrm{H}$, et al. (2009) Protein kinase and phosphatase signaling in Mycobacterium tuberculosis physiology and pathogenesis. Biochim Biophys Acta 1804: 620-627.

2. Deol P, Vohra R, Saini AK, Singh A, Chandra H, et al. (2005) Role of
Mycobacterium tuberculosis Ser/Thr kinase PknF: Implications in glucose transport and cell division. J Bacteriol 187: 3415-3420.

3. Kang CM, Abbott DW, Park ST, Dascher CC, Cantley LC, et al. (2005) The Mycobacterium tuberculosis serine/threonine kinases PknA and PknB: Substrate identification and regulation of cell shape. Genes Dev 19: 1692-1704.

4. Lakshminarayan H, Rajaram A, Narayanan S (2009) Involvement of serine threonine protein kinase, PknL, from Mycobacterium tuberculosis H37Rv in starvation response of mycobacteria. Journal of Microbial \& Biochemical Technology 1: 30-36.

5. Gopalaswamy R, Narayanan S, Chen B, Jacobs WR, Av-Gay Y (2009) The serine/threonine protein kinase Pknl controls the growth of Mycobacterium tuberculosis upon infection. FEMS Microbiol Lett 295: 23-29.

6. Jayakumar D, Jacobs WR, Narayanan S (2008) Protein kinase E of Mycobacterium tuberculosis has a role in the nitric oxide stress response and apoptosis in a human macrophage model of infection. Cell Microbiol 10: 365374.

7. Malhotra V, Arteaga-Cortes LT, Clay G, Clark-Curtiss JE (2010) Mycobacterium tuberculosis protein kinase $\mathrm{K}$ confers survival advantage during early infection in mice and regulates growth in culture and during persistent infection Implications for immune modulation. Microbiology 156: 2829-2841. 
Citation: Parandhaman DK, Hassan S, Narayanan S (2016) Multifaced pknE: Apoptosis Inhibition, HIV Co-Infection, Host Signaling Cross-Talk and in Orchestrating the Physiology of Mycobacterium tuberculosis. J Microb Biochem Technol 8: 231-235. doi: 10.4172/1948-5948.1000291

8. Papavinasasundaram KG, Chan B, Chung JH, Colston MJ, Davis EO, et al. (2005) Deletion of the Mycobacterium tuberculosis pknH gene confers a higher bacillary load during the chronic phase of infection in BALB/c mice. J Bacteriol 187: $5751-5760$.

9. Walburger A, Koul A, Ferrari G, Nguyen L, Prescianotto-Baschong C, et al. (2004) Protein kinase $G$ from pathogenic mycobacteria promotes survival within macrophages. Science 18: 1800-1804.

10. Cowley S, Ko M, Pick N, Chow R, Downing KJ, et al. (2004) The Mycobacterium tuberculosis protein serine/threonine kinase PknG is linked to cellular glutamate/glutamine levels and is important for growth in vivo. Mol Microbiol 52: 1691-1702.

11. Gopalaswamy R, Narayanan S, Jacobs WR Jr, Av-Gay Y (2008) Mycobacterium smegmatis biofilm formation and sliding motility are affected by the serine/ threonine protein kinase PknF. FEMS Microbiol Lett 278: 121-127.

12. Kumar D, Palaniyandi K, Challu VK, Kumar P, Narayanan S (2012) PknE, a serine/threonine protein kinase from Mycobacterium tuberculosis has a role in adaptive responses. Arch Microbiol 195: 75-80.

13. Av-Gay Y, Everett M (2000) The eukaryotic-like Ser/Thr protein kinases of Mycobacterium tuberculosis. Trends Microbiol 8: 238-244.

14. Molle V, Girard-Blanc C, Kremer L, Doublet P, Cozzone AJ, et al. (2003) Protein PknE, a novel transmembrane eukaryotic-like serine/threonine kinase from Mycobacterium tuberculosis. Biochem Biophys Res Commun 5: 820-825.

15. Gay LM, Ng HL, Alber T (2006) A conserved dimer and global conformational changes in the structure of apo-PknE Ser/Thr protein kinase from Mycobacterium tuberculosis. J Mol Biol 360: 409-420.

16. Narayan A, Sachdeva P, Sharma K, Saini AK, Tyagi AK, et al. (2007) Serine threonine protein kinases of mycobacterial genus: Phylogeny to function. Physiol Genomics. 29: 66-75.

17. Galkin AN, Mikheeva LE, Shestakov SV (2003) Insertional inactivation of genes encoding eukaryotic type serine/threonine protein kinases in cyanobacterium Synechocystis sp. PCC 6803. Mikrobiologiia 72: 64-69.

18. Zhang CC, Friry A, Peng L (1998) Molecular and genetic analysis of two closely linked genes that encode, respectively, a protein phosphatase $1 / 2 A / 2 B$ homolog and a protein kinase homolog in the cyanobacterium Anabaena sp. strain PCC 7120. J Bacteriol 180: 2616-2622.

19. Bardarov S, Bardarov S, Pavelka MS, Sambandamurthy V, Larsen M, et al. (2002) Specialized transduction: An efficient method for generating marked and unmarked targeted gene disruptions in Mycobacterium tuberculosis, M. bovis BCG and M. smegmatis. Microbiology 148: 3007-3017.

20. Parandhaman DK, Narayanan S (2014) Cell death paradigms in the pathogenesis of Mycobacterium tuberculosis infection. Front Cell Infect Microbiol 4: 31

21. Kumar D, Narayanan S (2012) pknE, a serine/threonine kinase of Mycobacterium tuberculosis modulates multiple apoptotic paradigms. Infect Genet Evol 12: 737-747.
22. Cargnello M, Roux PP (2011) Activation and function of the MAPKs and their substrates, the MAPK-activated protein kinases. Microbiol Mol Biol Rev 75 50-83.

23. Schorey JS, Cooper AM (2003) Macrophage signalling upon mycobacteria infection: The MAP kinases lead the way. Cell Microbiol 5: 133-142.

24. Parandhaman DK, Hanna LE, Narayanan S (2014) PknE, a serine/threonine protein kinase of Mycobacterium tuberculosis initiates survival cross-talk that also impacts HIV co-infection. PLoS One 9: e83541.

25. Jung JY, Madan-Lala R, Georgieva M, Rengarajan J, Sohaskey CD, et al. (2013) The intracellular environment of human macrophages that produce nitric oxide promotes growth of mycobacteria. Infect Immun 81: 3198-3209.

26. Blond D, Raoul H, Le Grand R, Dormont D (2000) Nitric oxide synthesis enhances human immunodeficiency virus replication in primary human macrophages. J Virol 74: 8904-8912.

27. Li H, Meininger CJ, Hawker JR, Haynes TE, Kepka-Lenhart D, et al. (2001) Regulatory role of arginase I and II in nitric oxide, polyamine and proline syntheses in endothelial cells. Am J Physiol Endocrinol Metab 280: E75-E82.

28. Kasmi KC, Qualls JE, Pesce JT, Smith AM, Thompson RW, et al. (2008) Tolllike receptor-induced arginase 1 in macrophages thwarts effective immunity against intracellular pathogens. Nat Immunol 9: 1399-1406.

29. Gobert AP, Cheng Y, Wang JY, Boucher JL, lyer RK, et al. (2002) Helicobacter pylori induces macrophage apoptosis by activation of arginase II. J Immunol 168: $4692-4700$.

30. Zumla A, Raviglione M, Hafner R, von Reyn CF (2013) Tuberculosis. N Engl J Med 368: 745-755.

31. Getahun H, Gunneberg C, Granich R, Nunn P (2010) HIV infection-associated tuberculosis: The epidemiology and the response. Clin Infect Dis 50 Suppl 3 S201-207.

32. Villarino A, Duran $R$, Wehenkel A, Fernandez $P$, England $P$, et al (2005) Proteomic identification of $M$. tuberculosis protein kinase substrates: PknB recruits GarA, a FHA domain-containing protein, through activation loopmediated interactions. J Mol Biol 29: 953-963.

33. Zheng X, Papavinasasundaram KG, Av-Gay Y (2007) Novel substrates of Mycobacterium tuberculosis PknH Ser/Thr kinase. Biochem Biophys Res Commun 355: 162-168.

34. Cui T, Zhang L, Wang X, He ZG (2009) Uncovering new signaling proteins and potential drug targets through the interactome analysis of Mycobacterium tuberculosis. BMC Genomics 10: 118.

35. Gurung AB, Mylliemngap BJ, Bhattacharjee A, Ali MA, Al-Hemaid FM (2015) Interactome analysis and design of inhibitors against selected protein targets of Ser/Thr protein kinase (STPK) signaling pathways in Mycobacterium tuberculosis H37Rv. Genet Mol Res 14: 10390-10403.

36. Parandhaman DK, Sharma P, Bisht D, Narayanan S (2014) Proteome and phosphoproteome analysis of the serine/threonine protein kinase $\mathrm{E}$ mutant of Mycobacterium tuberculosis. Life Sci 109: 116-126. 